\title{
The Role of Wharton's Jelly Mesenchymal Stem Cells in Skin Reconstruction
}

\author{
Ayoob Rostamzadeh "; Maryam Anjomshoa "; Soleiman Kurd ${ }^{2}$; Jia-Ke Chai ${ }^{3}$; Faeze \\ Jahangiri ${ }^{4}$; Mohammad Ali Nilforoushzadeh ${ }^{4,5,}$; Sona Zare ${ }^{4, *}$ \\ ${ }^{1}$ Department of Anatomical Sciences, Faculty of Medicine, Shahrekord University of Medical Sciences, Shahrekord, Iran \\ ${ }^{2}$ School of Advanced Technologies in Medicine, Shahid Beheshti University of Medical Sciences, Tehran, Iran \\ ${ }^{3}$ Department of Burn and Plastic Surgery, Burn and Plastic Hospital of PLA General Hospital, Beijing, China \\ ${ }^{4}$ Skin and Stem Cell Research Center, Tehran University of Medical Sciences, Tehran, Iran \\ 5 Skin Diseases and Leishmaniasis Research Center, Isfahan University of Medical Sciences, Isfahan, Iran \\ ${ }^{*}$ Corresponding authors: Sona Zare, Skin and Stem Cell Research Center, Tehran University of Medical Sciences, Tehran, Iran. Tel: +98-2122201710, Fax: +98-2122239264, \\ E-mail: sonazarebio@gmail.com; Mohammad Ali Nilforoushzadeh, Skin and Stem Cell Research Center, Tehran University of Medical Sciences, Tehran, Iran. Tel: +98-2122201710, \\ Fax: +98-2122239264, E-mail:dr_nilforoush@yahoo.com
}

Received: January 23, 2015; Revised: May 8, 2015; Accepted: May 30, 2015

\begin{abstract}
Context:Stem cell therapy, especially in the segment of mesenchymal stem cells (MSCs), is one of the most promising areas of regenerative medicine.

Evidence Acquisition: According to research conducted by various researchers, Wharton's Jelly mesenchymal stem cells (WJMSCs) have several advantages compared to others sources, in regenerative medicine: WJMSCs are more primary cells; WJMSCs can be easily isolated and without invasive procedures; WJMSCs have no ethical problems; WJMSCs are more cost effective than other sources of MSCs. Also, WJMSCs were demonstrated to express stem cell mesenchymal markers.

Results: Similar to bone marrow MSCs, WJMSCs express major histocompatibility complex (MHC) class I molecules.

Conclusions: Although the aforementioned challenges must still be addressed, the potential of WJMSCs in skin regenerative clinical treatments is promising.
\end{abstract}

Keywords: Wharton's Jelly; Mesenchymal Stem Cells; Skin; Cell- and Tissue-Based Therapy

\section{Context}

\subsection{What is Stem Cell?}

Stem cells are self-renewing and undifferentiated cells that have the potential to differentiate into various functional cell types (1-4). Self-renewal, the capacity to generate identical daughter cells, without any differentiation, is necessary to preserve stem cell pools in different tissues $(3,5)$.

\subsection{Stem Cells Classification}

\subsubsection{Classification on Source Origin}

Stem cells could be classified into two main types, based on their source of origin: Embryonic, which derived from the inner cell mass of preimplantation embryo and has the ability of constructing all three embryonic germ layers, and Adult, scattered in various tissues and organs, and has the capability of producing at least one type of differentiated functional progeny (4, 6-9). Although the latter were thought to have a limited capacity, recent evidence have attested an extensive capability to generate cells of the three different lineages, like the former (10-12).

\subsubsection{Classification on Potential of Differentiation}

Another possible classification for stem cells is based on their potential range of differentiation (3), which, in turn, are subtyped into totipotent, pluripotent, multipotent, and unipotent. Totipotent cells, such as zygote and early blastomers (1-3 days from oocyte fertilization) have the capacity to generate all types of cells, while pluripotent cells, such as inner cell mass of blastocysts (4-14 days after oocyte fertilization) could produce all cell types, except extraembryonic trophoblast lineage (3, 8, 12-14). Mouse and human oogonial stem cells express high levels of telomerase catalytic subunit, which is a hallmark feature of pluripotent and germ cells (15). Also, multilineage-differentiating stress-enduring (MUSE) cells are pluripotent cells that have ability to differentiate into cell types from all three germ layers, from a single cell (16). Multipotent stem cells have the ability to differentiate into all cell types, within one particular lineage $(13,14)$, while unipotent stem cells are defined as cells that have the competency of differentiating into only one lineage (3).

Copyright (C) 2015, Skin and Stem Cell Journal. This is an open-access article distributed under the terms of the Creative Commons Attribution-NonCommercial 4.0 International License (http://creativecommons.org/licenses/by-nc/4.0/) which permits copy and redistribute the material just in noncommercial usages, provided the original work is properly cited. 
Rostamzadeh A et al.

\section{Evidence Acquisition}

\subsection{Structural Properties and Characterization of Multipotent Stem Cells}

Multipotent stem cells (MSCs) have characteristics similar to others cell and have a significant role in development, tissue, defense, and repair, such as neural and cardiac disorders, which made them a familiar subject of study, with enormous productivity in the upcoming of medical sciences $(17,18)$. Although the MSCs could produce other lines of cells, there are several limitations in their differentiation capacity. For example, MSCs in the brain can differentiate into disparate neural cells and glia or hematopoietic stem cells, which have the capacity of differentiation into most of the blood cells. However, they do not have the ability to produce brain cells. Furthermore, bone marrow contains MSCs, which can differentiate into the whole types of blood cell (18). Due to the mentioned limitations, MSCs are classified as adult stem cells; the most well-known MSCs, namely mesenchymal stem cells have the ability to constitute various numbers of cells, such as bone, muscle, cartilage, fat, etc., under specific conditions $(19,20)$. The MSCs can fundamentally create particular cell types. These kinds of stem cells are different from pluripotent stem cells, which can produce nearly all cell types, or totipotent stem cells, which can differentiate into any cell (21). Pluripotent stem cells essentially specialize into MSCs, and formerly MSCs produce cells with a definite target and role $(17,19-22)$. In regard to the application of MSCs in treatment protocols, expression of chemokine receptors in these cells made them respond to the increasing amount of chemokines produced in injured tissue, and their further migration to the tissue of target (23). Several applications of MSCs in animal models are as follows: spinal cord injury, bone fracture, autoimmune disorders, rheumatoid arthritis, and hematopoietic defects (24-26). This area of research makes another approach in stem cell therapy; in this field, the cells are transplanted to another case, which represents a different allogeneic host, instead of "self". Even though this way of diseases management is still continuing, it is worth noting to protect tissues before organ transplantation. Besides their use in tissue regeneration, mesenchymal stem cells are employed for drug monitor (17). The MSCs have been considered as a patient-specific drugstore for injured tissue (27). Effortless differentiation ability and commitment made mesenchymal tissue cells a noticeable issue of discussion, in advance. Researchers initially supposed MSCs are recognized to derive as pericytes, which have the ability of surveying their domain, act as responder to limited stimulants with many useful interventions (28). Easy access and adaptation declare another beneficial aspect for using MSCs in therapeutic protocols (29). Recently, neural cells, as main forms of MSCs, are valued in research studies, owing to their differentiation potential into nerve cells, without revenue level, as it could be seen in other cells, such as blood. Their isolation possibility from adult and fetal brain tissues mean that these cells can differentiate into new nerve cells, which in turn makes them a suitable choice for brain and spinal cord injuries treatment $(17,30,31)$. Meanwhile, MSCs are separated from pluripotent stem cells; by now, these cells have incompletely differentiated with further specification during their growing. Multipotent adult stem cells seem mainly beneficial in transplantation, owing to easy harvesting, extensive expansion, and also, no immune rejection, owing to having immunomodulatory function and ethical problems (32). Some MSCs have trophic properties through expression of growth factors and chemokines for inducing cell proliferation and angiogenesis, and mitogenic proteins (for example, transforming tumor growth factor-alpha (TGF- $\alpha$ ); TGF- $\alpha$, TGF- $\beta$, hepatocyte growth factor (HGF), epithelial growth factor (EGF), basic fibroblast growth factor (bFGF) and insulin-like growth factor-1 (IGF-1) to enhance fibroblast, epithelial and endothelial cells division (33-35) and strength the endothelial cell line and set off angiogenesis, through vascular endothelial growth factor (VEGF), IGF-1, EGF andangiopoietin-1 (36). An anti-inflammatory action is one of the other benefits of MSC. The MSCs have compound response mechanisms amongst the different immune cells, through production of anti-inflammatory proteins in reaction to inflammatory molecules, including interleukin-1 (IL-1), IL-2, IL-12, tumor necrosis factor- $\alpha$ and interferon-gamma (37). Eventually, anti-apoptotic properties, by producing IGF-1 and IL-6 for up-regulation of Akt (protein kinase B) and nuclear factor kappa-light-chain-enhancer of activated B cells, may constitute another advantageous aspect for MSCs, as antiapoptotic agents $(17,38)$.

\subsection{Wharton's Jelly Multipotent Stem Cells}

The MSCs, under specific culture conditions, hold the ability to differentiate into a variety of lines, such as bone (39), skin (40), cartilage (41), adipose (42), endothelium (43), muscle (44), and neuronal cells (45). The MSCs have various sources, including adipose tissue, muscle, skin, bone marrow, Wharton's Jelly, and synovial joint (46). Mitchell et al. (2003) differentiates MSCs of Wharton's Jelly (WJMSCs) in low serum medium, containing FGF into neurons and glial cells (47). Troyer and Weiss (2008), while studying the immunological properties, found that transplantation rejection of undifferentiated human WJMSCs (h-WJMSCs) was not possible (48). Human WJMSCs could be induced to form other cell lines, such as neurons and glial cells (47), germ like cells (49), endothelial (50) and insulin secreting cells (51). Regarding bone marrow mesenchymal stem cells (BMMSCs), due to reduced differentiation potential of MSCs, with increasing age of the donor, researchers seek other sources of MSCs, including WJMSCs. According to investigations conducted by various researchers, WJMSCs have advantages compared to others sources: 1) WJMSCs are more primary cells; 2) WJMSCs can be easily isolated and with- 
out invasive procedures, 3) WJMSCs have no ethical problem; 4) WJMSCs are more cost effective than other sources of MSCs (52). The WJMSCs express stem cell mesenchymal markers (CD44, CD105), whereas they do not express the hematopoietic markers (CD34, CD45) (53). Similar to bone marrow MSCs, WJMSCs express major histocompatibility complex (MHC) class I molecules (such as human leukocyte antigen (HLA)-A, -B, -C), while they do not express MHC class II (HLA-DR) on their cell surface $(54,55)$. In recent years, MSCs derived from umbilical cord matrix Wharton's Jelly have attracted much interest. Wharton's Jelly is a mature mucous tissue and the main component of the umbilical cord, connecting the umbilical vessels to the amniotic epithelium. Umbilical cord derives from extra-embryonic or embryonic mesoderm; at birth, it weighs about $40 \mathrm{~g}$ and measures approximately 30-65 $\mathrm{cm}$ in length and $1.5 \mathrm{~cm}$ in width (56). Individual differences are observed within newborn babies. Fong and colleagues characterized Wharton's Jelly stem cells and found the presence of both embryonic and MSCs, targeting this source, as unique and of valuable use for clinical applications. The WJMSCs can be cultured with little or even no major loss trough at least 50 passages, poor capability to differentiate into adipocytes, shorter doubling times, compared to BMMSCs (57). The WJMSCs are derived from the cushioning matrix between the umbilical blood vessels, rather than from umbilical blood (58). The cluster of differentiation 73 (CD73), CD90, CD105 and HLA class I are positive, whereas CD45, CD34 and HLA class II are negative. Researchers confirmed them to differentiate into endothelial cells (59) after the addition of VEGF and bFGF to cultures, which are critical to cutaneous repair.

\subsection{Wharton's Jelly Multipotent Stem Cells and Skin Reconstruction}

The MSCs have been shown to possess a strong ability to improve tissue damage in response to skin injury, by contributing to collagen deposition (60), wound contraction (61), angiogenesis (62), regeneration of skin appendages, and enhanced growth of epidermal cells (63). Currently, most of the studies related to MSCs in clinical treatments are on BMMSCs; only limited studies mentioned the application of WJMSCs. However, the process of obtaining the umbilical cord-derived MSCs is much easier and does not harm the donor, compared with that of BMMSCs. Similar to BMMSCs, MSCs derived from Wharton's Jelly can adapt to the culture conditions of the dermal equivalents (DEs), suggesting that WJMSCs confer a therapeutic benefit by supporting the regeneration of the dermal compartment (40). Interestingly, a small population of WJMSCs coexpresses the mesenchymal marker vimentin and the epithelial marker pan-cytokeratin (CK). By contrast, BMMSCs are CK-negative after isolation and on DEs. Compared with BMMSCs, WJMSCs can survive on DEs and can adapt more easily to the culture conditions of the skin. Therefore, WJMSCs can promote skin epithelization, vasculogenesis, and collagen deposition, by secreting a number of soluble factors, during the wound healing phase. Therefore, WJMSCs can be used as an interesting and promising tool to regenerate skin wounds. The restoration of cutaneous appendages, after a severe skin injury, which is related to the function of the regenerated skin and affects the quality of life, may be an important function of WJMSCs during skin repair. The regeneration of sweat glands, after deep burns, has been an unresolved challenge. To address this problem, our group previously induced BMMSCs to acquire the phenotype of sweat gland cells in vitro and then transplanted them into fresh skin wounds, made by excising the anhidrotic scars of five patients after their deep burn injuries were healed (64). The MSCs transformed into sweat gland cells and facilitated the recovery of functional sweat glands. This phenomenon may help address the problem of sweat gland depletion in patients surviving extensive deep burns (64). The WJMSCs, in a specific induction system, may hopefully differentiate into sweat gland cell-like cells. Recently, WJMSCs were successfully induced to differentiate into sweat gland cells in vitro (65). Studies indicated that WJMSCs can differentiate into sweat gland-like cells, via a novel and feasible system that is more effective than our previous method (64). The WJMSCs, as a novel source of stem cells, can differentiate into sweat glands, for further regeneration of the epidermis and skin appendages.

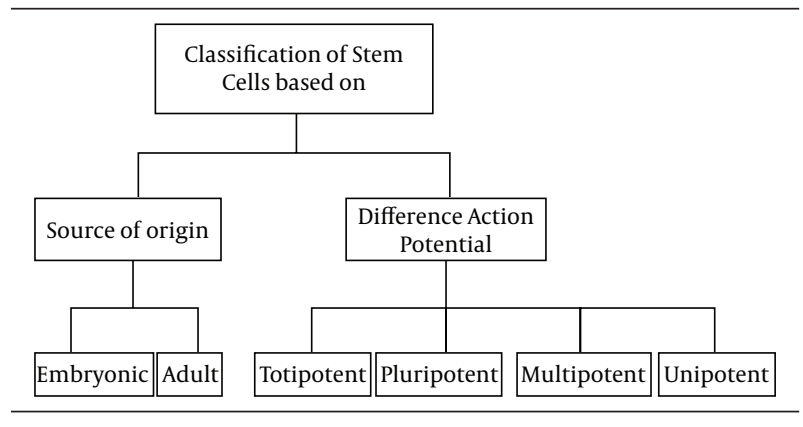

Figure 1. Stem Cells Classification

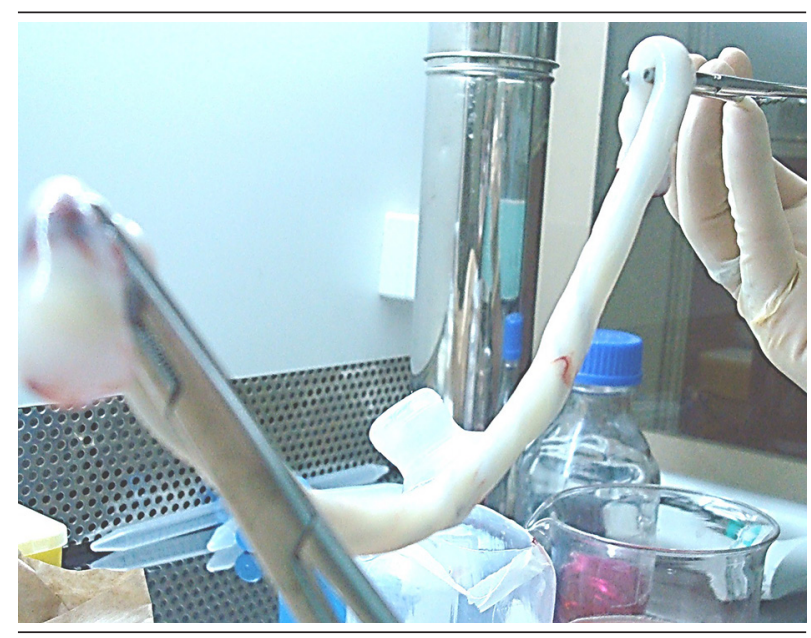

Figure 2. Umbilical Cord 

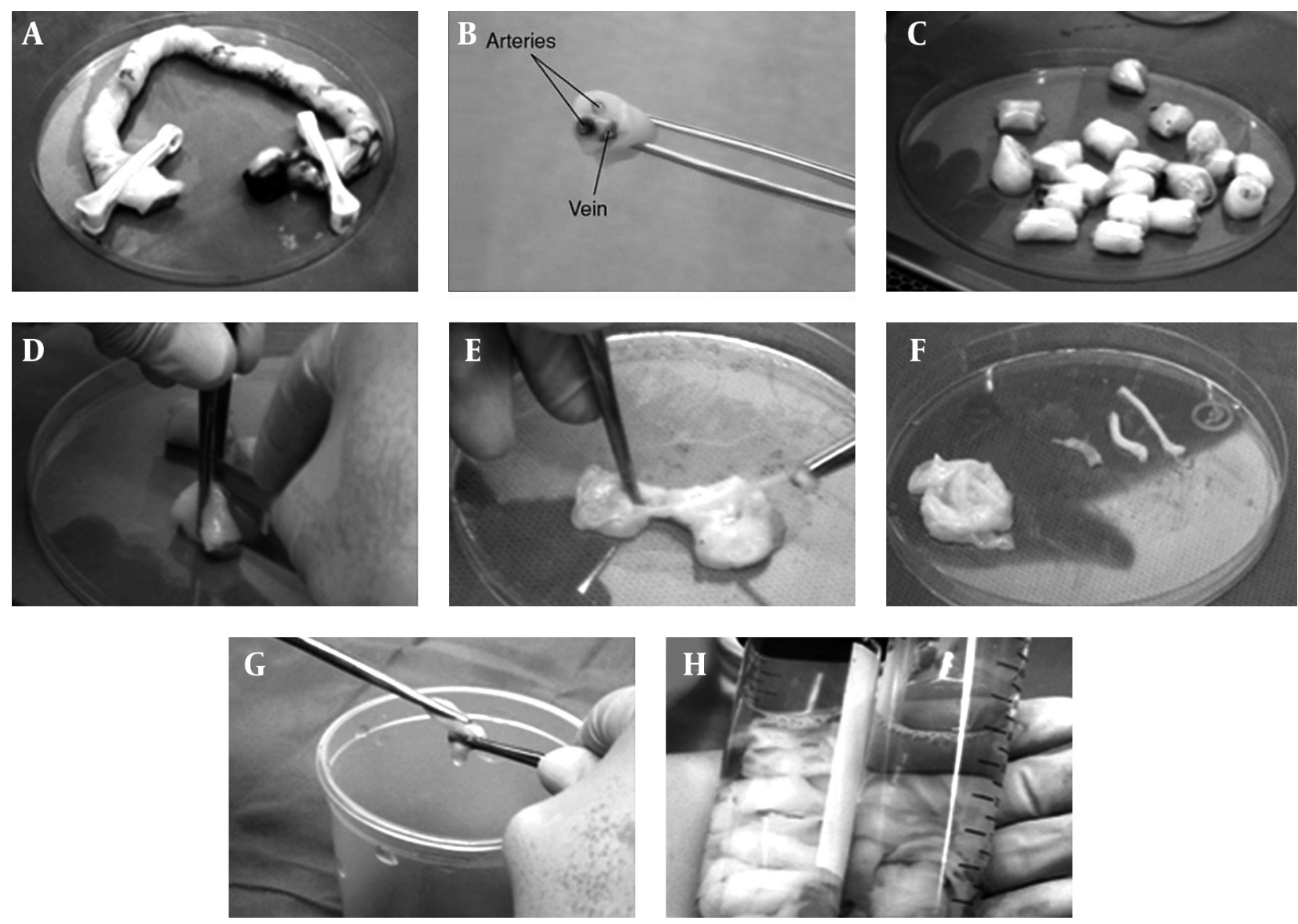

Figure 3. Isolation and Culture of Wharton's Jelly Mesenchymal Stem Cells From Umbilical Cord

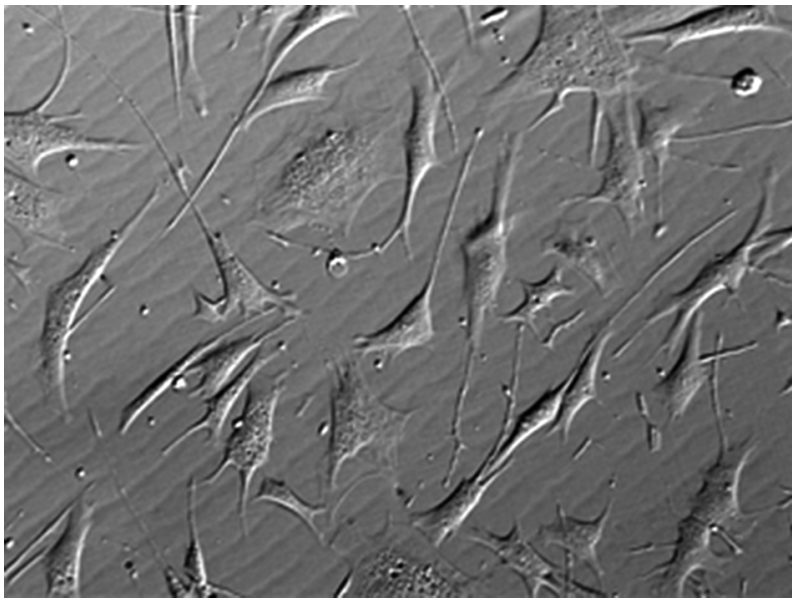

Figure 4. Primary Wharton's Jelly Mesenchymal Stem Cells Culture

\section{Results}

As a challenge that WJMSC transplantation has not yet been approved by the Food and Drug Administration and that clinical trials of MSC transplantation, showing no adverse events, have not yet reached the 10-year mark,
WJMSCs have not been extensively used for clinical therapies. In addition, a number of challenges and side effects in WJMSC-based therapy remain to be addressed. First, the umbilical cord is an allogeneic tissue for the recipient. The second problem is an ethical one. Although the umbilical cord is a disused organ, both the donor and the recipient have the right of informed consent. Third, a complete detection system, to test any potential disease risk in donors, has yet to be established. Fourth, the isolation and further expansion of primitive MSCs probably have several uncertain factors, such as the use of animal serum and the most common culture method in vitro, which should be avoided in clinical-grade therapy to prevent cell contact with xenogeneic proteins. Fifth, potential long term risks associated with MSC therapy that may not have been observable within a short period, after administrations have recently been confirmed in preclinical studies. These risks include potential maldifferentiation, immunosuppression and malignant tumor growth, which are the primary safety concern, as well as the promotion of tumor growth, when the treatment was systemically administered into animals with coexisting malignancy $(66,67)$. Lastly, as products of cell therapy, product consistency, cell stability, and toxicity should also be considered. 


\section{Conclusions}

Stem cell therapy is one of the most promising areas of regenerative medicine, and a number of such therapies are now under development by academic and industrial groups. As the technology of cell therapies has advanced, regulatory systems, to monitor their use, must be established. The general strategy for many adult stem cells and all pluripotent stem cell therapies is the scale up of undifferentiated stem cell production. After differentiation into a specific cell type and delivery to patients, the cells may reside indefinitely. Given that the signal pathway for MSC recruitment and repair is not clear, future studies need to be conducted to strengthen the mechanism and establish a series of criteria for the donor and the recipient. Furthermore, MSC banking, which can support the successful establishment of umbilical cord blood banking, should be set up. Although the aforementioned challenges must still be addressed, the potential of WJMSCs in skin regenerative clinical treatments is promising.

\section{Authors' Contributions}

\section{All authors participated equally in this study.}

\section{Funding/Support}

This study was conducted by financial support of Skin and Stem Cell Research Center, Tehran University of Medical Sciences, Tehran, Iran.

\section{References}

1. Bongso A, Fong C. Human embryonic stem cells: their nature, properties, and uses. Trends in Stem Cell Biology and Technology: Springer; 2009. pp.1-17.

2. Sundelacruz S, Kaplan DL. Stem cell- and scaffold-based tissue engineering approaches to osteochondral regenerative medicine. Semin Cell Dev Biol. 2009;20(6):646-55.

3. Simón C, Pellicer A. Stem cells in human reproduction: basic science and therapeutic potential.: CRC Press; 2009.

4. Alison MR, Poulsom R, Forbes S, Wright NA. An introduction to stem cells.J Pathol. 2002;197(4):419-23.

5. Smith A. The battlefield of pluripotency. Cell. 2005;123(5):757-60.

6. Fu RH, Wang YC, Liu SP, Huang CM, Kang YH, Tsai CH, et al. Differentiation of stem cells: strategies for modifying surface biomaterials. Cell Transplant. 2011;20(1):37-47.

7. Weissman IL. Stem cells-scientific, medical, and political issues. N Engl J Med. 2002;346(20):1576-9.

8. Li L, Xie T. Stem cell niche: structure and function. Annu Rev Cell Dev Biol. 2005;21:605-31.

9. Nandedkar T, Narkar M. Stem cell research: its relevance to reproductive biology. Indian J Exp Biol. 2003;41(7):724-39.

10. Bunnell BA, Flaat M, Gagliardi C, Patel B, Ripoll C. Adipose-derived stem cells: isolation, expansion and differentiation. Methods. 2008;45(2):115-20.

11. Yoshida Y, Yamanaka S. iPS cells: a source of cardiac regeneration. J Mol Cell Cardiol. 2011;50(2):327-32.

12. Niibe K, Kawamura Y, Araki D, Morikawa S, Miura K, Suzuki S, et al. Purified mesenchymal stem cells are an efficient source for iPS cell induction. PLoS One. 2011;6(3):e17610.

13. Jaenisch R, Young R. Stem cells, the molecular circuitry of pluripotency and nuclear reprogramming. Cell. 2008;132(4):567-82.

14. Krampera M, Franchini M, Pizzolo G, Aprili G. Mesenchymal stem cells: from biology to clinical use. Blood Transfus. 2007;5(3):120-9.

15. Woods DC, White YA, Tilly JL. Purification of oogonial stem cells from adult mouse and human ovaries: an assessment of the literature and a view toward the future. Reprod Sci. 2013;20(1):7-15.

16. Wakao S, Kitada M, Kuroda Y, Shigemoto T, Matsuse D, Akashi H, et al. Multilineage-differentiating stress-enduring (Muse) cells are a primary source of induced pluripotent stem cells in human fibroblasts. Proc Natl Acad Sci U S A. 2011;108(24):9875-80.

17. Murphy MB, Moncivais K, Caplan AI. Mesenchymal stem cells: environmentally responsive therapeutics for regenerative medicine. Exp Mol Med. 2013;45:e54.

18. Mundra V, Gerling IC, Mahato RI. Mesenchymal stem cell-based therapy. Mol Pharm. 2013;10(1):77-89.

19. Bibber B, Sinha G, Lobba AR, Greco SJ, Rameshwar P. A review of stem cell translation and potential confounds by cancer stem cells. Stem Cells Int. 2013;2013:241048.

20. Campagnoli C, Roberts IA, Kumar S, Bennett PR, Bellantuono I, Fisk NM. Identification of mesenchymal stem/progenitor cells in human first-trimester fetal blood, liver, and bone marrow. Blood. 2001;98(8):2396-402.

21. Chen FM, Wu LA, Zhang M, Zhang R, Sun HH. Homing of endogenous stem/progenitor cells for in situ tissue regeneration: Promises, strategies, and translational perspectives. Biomaterials. 2011;32(12):3189-209.

22. He Q, Wan C, Li G. Concise review: multipotent mesenchymal stromal cells in blood. Stem Cells. 2007;25(1):69-77.

23. Friedenstein AJ, Chailakhyan RK, Latsinik NV, Panasyuk AF, Keiliss-Borok IV. Stromal cells responsible for transferring the microenvironment of the hemopoietic tissues. Cloning in vitro and retransplantation in vivo. Transplantation. 1974;17(4):331-40.

24. Weiss DJ, Bertoncello I, Borok Z, Kim C, Panoskaltsis-Mortari A, Reynolds S, et al. Stem cells and cell therapies in lung biology and lung diseases. Proc Am Thorac Soc. 2011;8(3):223-72.

25. Yagi H, Soto-Gutierrez A, Kitagawa Y, Tilles AW, Tompkins RG, Yarmush ML. Bone marrow mesenchymal stromal cells attenuate organ injury induced by LPS and burn. Cell Transplant. 2010;19(6):823-30.

26. Guan XJ, Song L, Han FF, Cui ZL, Chen X, Guo XJ, et al. Mesenchymal stem cells protect cigarette smoke-damaged lung and pulmonary function partly via VEGF-VEGF receptors. J Cell Biochem. 2013;114(2):323-35.

27. Caplan AI, Correa D. The MSC: an injury drugstore. Cell Stem Cell. 2011;9(1):11-5.

28. Caplan AI. Adult mesenchymal stem cells for tissue engineering versus regenerative medicine. J Cell Physiol. 2007;213(2):341-7.

29. Haynesworth SE, Baber MA, Caplan AI. Cell surface antigens on human marrow-derived mesenchymal cells are detected by monoclonal antibodies. Bone. 1992;13(1):69-80.

30. Butti E, Cusimano M, Bacigaluppi M, Martino G. Neurogenic and non-neurogenic functions of endogenous neural stem cells. Front Neurosci. 2014;8:92.

31. Kennea NL, Mehmet H. Neural stem cells. J Pathol. 2002;197(4):536-50.

32. Zuk PA, Zhu M, Ashjian P, De Ugarte DA, Huang JI, Mizuno H, et al. Human adipose tissue is a source of multipotent stem cells. Mol Biol Cell. 2002;13(12):4279-95.

33. Haynesworth SE, Baber MA, Caplan AI. Cytokine expression by human marrow-derived mesenchymal progenitor cells in vitro: effects of dexamethasone and IL-1 alpha. J Cell Physiol. 1996;166(3):585-92.

34. Doorn J, van de Peppel J, van Leeuwen JP, Groen N, van Blitterswijk CA, de Boer J. Pro-osteogenic trophic effects by PKA activation in human mesenchymal stromal cells. Biomaterials. 2011;32(26):6089-98.

35. Caplan AI, Bruder SP. Mesenchymal stem cells: building blocks for molecular medicine in the 21st century. Trends Mol Med. 2001;7(6):259-64.

36. Chen L, Tredget EE, Wu PY, Wu Y. Paracrine factors of mesenchymal stem cells recruit macrophages and endothelial lineage cells and enhance wound healing. PLoS One. 2008;3(4):e1886.

37. Aggarwal S, Pittenger MF. Human mesenchymal stem cells modulate allogeneic immune cell responses. Blood. 2005;105(4):1815-22.

38. Gnecchi M, He H, Noiseux N, Liang OD, Zhang L, Morello F, et al. Evidence supporting paracrine hypothesis for Akt-modified 
mesenchymal stem cell-mediated cardiac protection and functional improvement. FASEB J. 2006;20(6):661-9.

39. Lennon DP, Edmison JM, Caplan AI. Cultivation of rat marrowderived mesenchymal stem cells in reduced oxygen tension: effects on in vitro and in vivo osteochondrogenesis.J Cell Physiol. 2001;187(3):345-55.

40. Schneider RK, Pullen A, Kramann R, Bornemann J, Knuchel R, Neuss $\mathrm{S}$, et al. Long-term survival and characterisation of human umbilical cord-derived mesenchymal stem cells on dermal equivalents. Differentiation. 2010;79(3):182-93.

41. Ringe J, Häupl T, Sittinger M. [Mesenchymal stem cells for tissue engineering of bone and cartilage]. Medizinische Klinik (Munich, Germany: 1983). 2003;98:35-40.

42. Dominici M, Le Blanc K, Mueller I, Slaper-Cortenbach I, Marini F, Krause D, et al. Minimal criteria for defining multipotent mesenchymal stromal cells. The International Society for Cellular Therapy position statement. Cytotherapy. 2006;8(4):315-7.

43. Silva GV, Litovsky S, Assad JA, Sousa AL, Martin BJ, Vela D, et al. Mesenchymal stem cells differentiate into an endothelial phenotype, enhance vascular density, and improve heart function in a canine chronic ischemia model. Circulation. 2005;111(2):150-6.

44. Makino S, Fukuda K, Miyoshi S, Konishi F, Kodama H, Pan J, et al. Cardiomyocytes can be generated from marrow stromal cells in vitro.J Clin Invest. 1999;103(5):697-705.

45. Lei Z, Yongda L, Jun M, Yingyu S, Shaoju Z, Xinwen Z, et al. Culture and neural differentiation of rat bone marrow mesenchymal stem cells in vitro. Cell Biol Int. 2007;31(9):916-23.

46. Wang Y, Nan X, Li Y, Zhang R, Yue W, Yan F, et al. Induction of umbilical cord blood-derived beta2m-c-Met+ cells into hepatocyte-like cells by coculture with CFSC/HGF cells. Liver Transpl. 2005;11(6):635-43.

47. Mitchell KE, Weiss ML, Mitchell BM, Martin P, Davis D, Morales L, et al. Matrix cells from Wharton's jelly form neurons and glia. Stem Cells. 2003;21(1):50-60.

48. Troyer DL, Weiss ML. Wharton's jelly-derived cells are a primitive stromal cell population. Stem Cells. 2008;26(3):591-9.

49. Huang P, Lin LM, Wu XY, Tang QL, Feng XY, Lin GY, et al. Differentiation of human umbilical cord Wharton's jelly-derived mesenchymal stem cells into germ-like cells in vitro. J Cell Biochem. 2010;109(4):747-54.

50. Chen MY, Lie PC, Li ZL, Wei X. Endothelial differentiation of Wharton's jelly-derived mesenchymal stem cells in comparison with bone marrow-derived mesenchymal stem cells. Exp Hematol. 2009;37(5):629-40.

51. Wu LF, Wang NN, Liu YS, Wei X. Differentiation of Wharton's jelly primitive stromal cells into insulin-producing cells in comparison with bone marrow mesenchymal stem cells. Tissue Eng Part A. 2009;15(10):2865-73.

52. Angelucci S, Marchisio M, Di Giuseppe F, Pierdomenico L, Sulpizio M, Eleuterio E, et al. Research Proteome analysis of human Wharton's jelly cells during in vitro expansion. Promote Sci. 2010;8:18.

53. Sakamoto T, Ono H, Saito Y. [Electron microscopic histochemical studies on the localization of hyaluronic acid in Wharton's jelly of the human umbilical cord]. Nihon Sanka Fujinka Gakkai Zasshi. 1996;48(7):501-7.

54. Turnovcova K, Ruzickova K, Vanecek V, Sykova E, Jendelova P. Properties and growth of human bone marrow mesenchymal stromal cells cultivated in different media. Cytotherapy. 2009;11(7):874-85.

55. Weiss ML, Medicetty S, Bledsoe AR, Rachakatla RS, Choi M, Merchav S, et al. Human umbilical cord matrix stem cells: preliminary characterization and effect of transplantation in a rodent model of Parkinson's disease. Stem Cells. 2006;24(3):781-92.

56. Conconi MT, Di Liddo R, Tommasini M, Calore C, Parnigotto PP. Phenotype and differentiation potential of stromal populations obtained from various zones of human umbilical cord: An overview. Open Tissue Eng Regen Med J. 2011;4(1):6-20.

57. Fong CY, Richards M, Manasi N, Biswas A, Bongso A. Comparative growth behaviour and characterization of stem cells from human Wharton's jelly. Reprod Biomed Online. 2007;15(6):708-18.

58. Kalaszczynska I, Ferdyn K. Wharton's jelly derived mesenchymal stem cells: future of regenerative medicine? Recent findings and clinical significance. Biomed Res Int. 2015;2015:430847.

59. Wu KH, Zhou B, Lu SH, Feng B, Yang SG, Du WT, et al. In vitro and in vivo differentiation of human umbilical cord derived stem cells into endothelial cells.J Cell Biochem. 2007;100(3):608-16.

60. Fathke C, Wilson L, Hutter J, Kapoor V, Smith A, Hocking A, et al Contribution of bone marrow-derived cells to skin: collagen deposition and wound repair. Stem Cells. 2004;22(5):812-22.

61. Neuss S, Schneider RK, Tietze L, Knuchel R, Jahnen-Dechent W. Secretion of fibrinolytic enzymes facilitates human mesenchymal stem cell invasion into fibrin clots. Cells Tissues Organs. 2010;191(1):36-46.

62. Halkos ME, Zhao ZQ, Kerendi F, Wang NP, Jiang R, Schmarkey LS, et al. Intravenous infusion of mesenchymal stem cells enhances regional perfusion and improves ventricular function in a porcine model of myocardial infarction. Basic Res Cardiol. 2008;103(6):525-36.

63. Nakagawa H, Akita S, Fukui M, Fujii T, Akino K. Human mesen chymal stem cells successfully improve skin-substitute wound healing. BrJ Dermatol. 2005;153(1):29-36.

64. Sheng Z, Fu X, Cai S, Lei Y, Sun T, Bai X, et al. Regeneration of functional sweat gland-like structures by transplanted differentiated bone marrow mesenchymal stem cells. Wound Repair Regen. 2009;17(3):427-35.

65. Tang M, Weir MD, Xu HH. Mannitol-containing macroporous calcium phosphate cement encapsulating human umbilical cord stem cells.J Tissue Eng Regen Med. 2012;6(3):214-24.

66. Klopp AH, Spaeth EL, Dembinski JL, Woodward WA, Munshi A, Meyn RE, et al. Tumor irradiation increases the recruitment of circulating mesenchymal stem cells into the tumor microenvironment. Cancer Res. 2007;67(24):11687-95.

67. Karnoub AE, Dash AB, Vo AP, Sullivan A, Brooks MW, Bell GW, et al. Mesenchymal stem cells within tumour stroma promote breast cancer metastasis. Nature. 2007;449(7162):557-63. 